\title{
MULTI-TASK DEEP LEARNING FOR SATELLITE IMAGE PANSHARPENING AND SEGMENTATION
}

\author{
Andrew Khalel ${ }^{1,2}$, Onur Tasar ${ }^{1}$, Guillaume Charpiat $^{3}$, Yuliya Tarabalka ${ }^{1}$ \\ ${ }^{1}$ TITANE team, Inria Sophia Antipolis - Méditerranée; ${ }^{2}$ Cairo University; ${ }^{3}$ TAU team, LRI, Inria Saclay \\ Email: firstname.name@inria.fr
}

\begin{abstract}
In this work, we propose a novel multi-task framework, to learn satellite image pansharpening and segmentation jointly. Our framework is based on the encoder-decoder architecture, where both tasks share the same encoder but each one has its own decoder. We compare our framework against singletask models with different architectures. Results show that our framework outperforms all other approaches in both tasks.
\end{abstract}

Index Terms - segmentation, pansharpening, multi-task, joint learning

\section{INTRODUCTION}

Recently, deep learning methods succeeded in getting detailed segmentation maps from aerial images. However, when applying deep learning methods to images acquired by Earth observing satellites, the resulting segmentation maps are often not very precise. This is partially due to the fact that most satellite imaging sensors capture separately a panchromatic image PAN (which has a high spatial resolution but consists only of a single band) and a multispectral image MS (which has more spectral bands but with lower spatial resolution). Most approaches start by creating a high resolution multispectral image HRMS, by "pansharpening", i.e. merging the PAN and MS images. All further analysis, such as segmentation or detection, is then performed on this pansharpened image; therefore the quality of the pansharpening process is crucial.

Pansharpening methods can be divided into two categories: classical and learning-based. The classical methods are usually generic statistical methods that can be applied to images from any satellite, without specific parameters. Some examples are: Bayesian fusion [1] and Local Mean and Variance Matching (LMVM) [2]. On the other hand, learning-based methods can tune parameters to each scenario to achieve better results. For instance, a three-layer network architecture (proposed earlier for super resolution) is used in [3] for pansharpening. PanNet [4] utilizes pansharpening domain knowledge by processing input data in a high pass domain instead of the image domain.

Semantic segmentation is the process of labeling each pixel of an image. Deep neural networks took over all classical methods in this field due to their excellent performance.
Convolutional neural networks are used in [5] to segment aerial images. U-nets, initially introduced for biomedical image segmentation [6], prove to achieve good results on remote sensing images too [7]. Finally, FuseNet architectures directly segment MS and PAN images with no need of pansharpening [8].

Multi-task learning (MTL) consists in training for several tasks simultaneously. Learning a single task can be very efficient and concentrated; however, the knowledge gained by the model during training does not guarantee to generalize well to new data, whereas developing features useful for several different tasks helps in picking more robust ones. Multi-task deep learning is usually implemented by hard or soft sharing of intermediate layer parameters. Hard parameter sharing consists in using the same layers for different tasks. It is the most common method and it has been proved to reduce overfitting risk significantly [9]. On the contrary, in soft parameter sharing, each task has its own model, and model similarity is encouraged by penalizing the distance between the parameters of each model.

In this paper, we introduce a framework that uses MTL to learn pansharpening and segmentation tasks jointly from MS and PAN images.

\section{METHODOLOGY}

We consider a single-encoder multi-decoder architecture, as shown in Fig. 1. A common encoder takes panchromatic (PAN) and multispectral (MS) images as input, and finds a data representation that satisfies all tasks. Each task is solved by a different decoder, plugged after the common encoder. The whole system is trained jointly from end to end.

The encoder consists of 4 convolution blocks, where each block comprises twice the sequence: convolution / batchnormalization / ReLU. Each convolution filter is of size $3 \times 3$. In the encoder, the MS image goes through a convolution block, and gets concatenated with the output of the PAN branch's second block, which has then the same resolution (thanks to the different number of maxpoolings in the two branches, see Fig. 11).

The encoder and the decoders are connected with a convolution block with 512 filters. Each task has a separate de- 


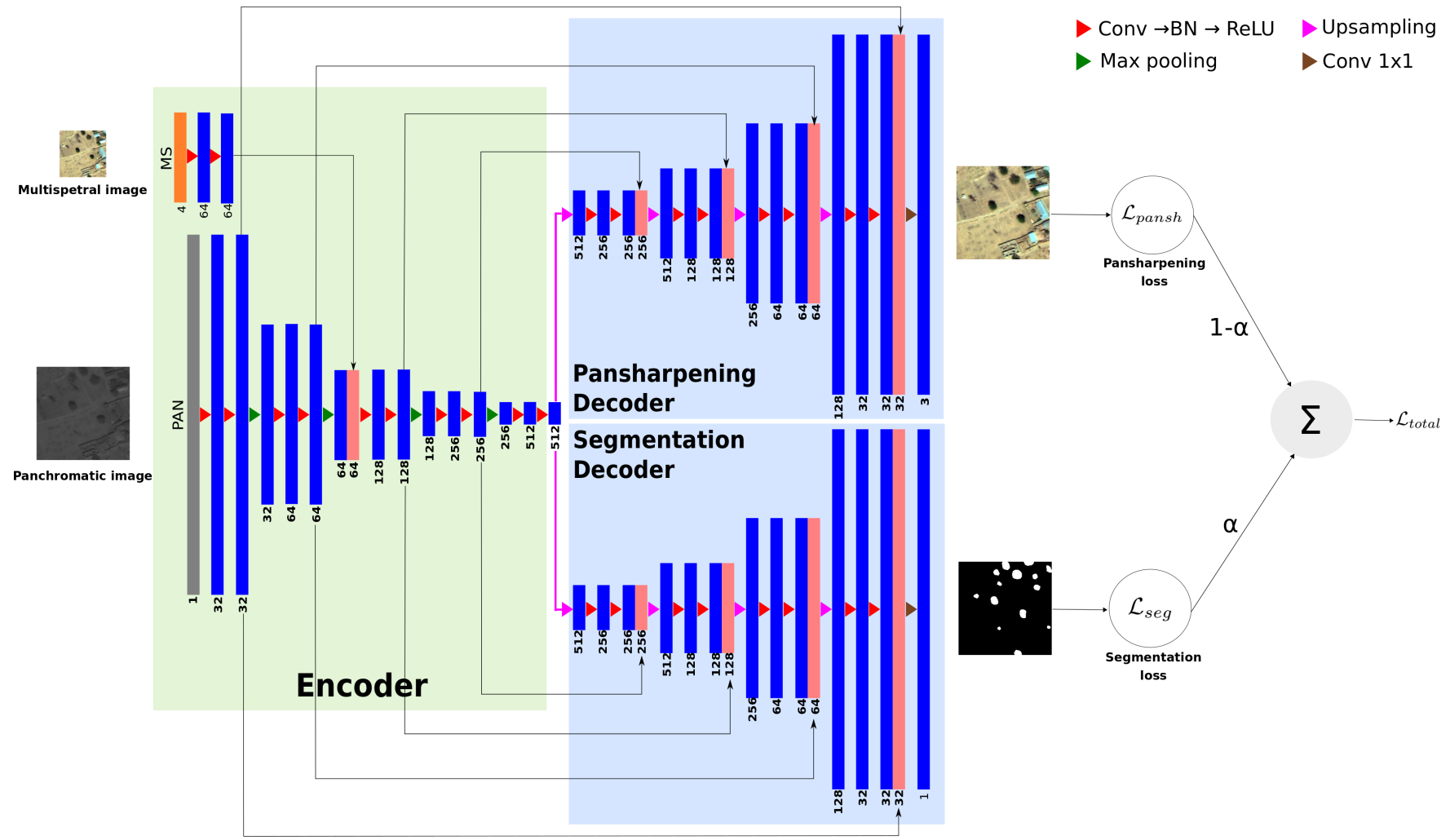

Fig. 1: The overall pansharpening and segmentation framework.

coder. The architecture of all decoders is the same: the one of the encoder's PAN branch, but in reverse order. At the end of each decoder, a $1 \times 1$ convolution layer is added, with a sigmoid activation. Finally, skip connections are added from each block of the encoder to the ones with similar resolution in the decoders.

Our loss function is a weighted sum of a segmentation loss $\left(\mathcal{L}_{\text {seg }}\right)$ and a pansharpening loss $\left(\mathcal{L}_{\text {pansh }}\right)$ :

$$
\mathcal{L}_{\text {total }}=\alpha \mathcal{L}_{\text {seg }}+(1-\alpha) \mathcal{L}_{\text {pansh }}
$$

where $\alpha \in[0,1]$ adjusts the relative importance of $\mathcal{L}_{\text {seg }}$ and $\mathcal{L}_{\text {pansh }}$. The segmentation loss is defined as:

$$
\mathcal{L}_{\text {seg }}=H-\log (J)
$$

where $H$ is the cross-entropy between ground-truth $\left(y_{i}\right)$ and predicted $\left(\hat{y}_{i}\right)$ pixel class probabilities, averaged over all $n$ pixels $i$ :

$$
H(\mathbf{y}, \hat{\mathbf{y}})=-\frac{1}{n} \sum_{i=1}^{n} y_{i} \log \left(\hat{y}_{i}\right)+\left(1-y_{i}\right) \log \left(1-\hat{y}_{i}\right)
$$

and $J$ is the soft intersection over union loss [10]:

$$
J(\mathbf{y}, \hat{\mathbf{y}})=\frac{\sum_{i} y_{i} \hat{y}_{i}}{\sum_{i} y_{i}+\hat{y}_{i}-y_{i} \hat{y}_{i}} .
$$

The pansharpening loss $\mathcal{L}_{\text {pansh }}$ is defined as a combination of the Universal Image Quality Index (Q) [11] and of the Peak Signal-to-Noise Ratio (PSNR):

$$
\mathcal{L}_{\text {pansh }}=(1-Q(\mathbf{y}, \hat{\mathbf{y}}))+\left(1-\frac{\operatorname{PSNR}(\mathbf{y}, \hat{\mathbf{y}})}{50}\right) .
$$

Since the maximum value of $Q$ is 1 , we normalized PSNR by a typical value, to enforce fairness in optimization. The source code of this work is publicly available on github 1 .

\section{EXPERIMENTS}

Our datase 12 consists of 25 images, each covering an area of $1 \mathrm{~km}^{2}$. These 11-bits images have been captured by the WorldView-3 satellite, with panchromatic and multispectral sensors of $0.31 \mathrm{~m}$ and $1.24 \mathrm{~m}$ resolution, respectively. The dataset has PAN, MS and HRMS images, together with segmentation masks for trees. Samples from the dataset are shown on Fig. 2.

We split our dataset into two sets: training set $(75 \%)$ and validation set $(25 \%)$. We took the top left quarter of each image as validation set, and the rest for training. To evaluate performance, Intersection over Union (IoU) [12] is used

1 https://github.com/andrewekhalel/MTL_PAN_SEG ${ }^{2}$ Dstl Satellite Imagery Feature Detection - https://www.kaggle. com/c/dstl-satellite-imagery-feature-detection 


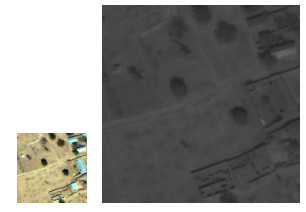

(a) MS

(b) PAN

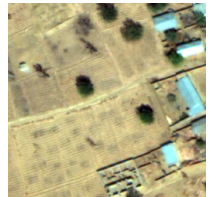

(c) HRMS

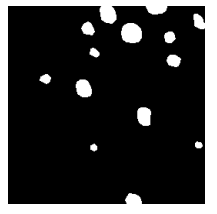

(d) Mask
Fig. 2: Example images from the WorldView-3 dataset.

for segmentation. Universal Image Quality Index (Q) [11], Peak noise-to-signal ratio (PSNR) and Erreur Relative Globale Adimensionnelle de Synthèse (ERGAS) [13] are used for pansharpening evaluation.

Table 1 shows the experimental setup parameters. During training and testing, data augmentation is used, to yield more robust models. The transformations used are: rotations (with angles 90, 180 and 270) and flips (horizontally and vertically).

\begin{tabular}{|c|c|}
\hline hyper-parameter & value \\
\hline \hline optimizer & Adam \\
\hline initial learning rate & $10^{-3}$ \\
\hline decay rate & 0.1 \\
\hline decay epoch & $10^{t h}$ \\
\hline batch size & 16 \\
\hline patch size & $256 \times 256$ \\
\hline epochs & 40 \\
\hline iterations per epoch & 5000 \\
\hline
\end{tabular}

Table 1: Parameter values.

We run a set of experiments to optimize the value of the weighing factor $\alpha$ in Eq. 11. Each experiment uses a different value for $\alpha$, starting from 0.1 to 0.9 with 0.1 linear step. To avoid fluctuations in the results (caused by weights initialization), we perform each experiment 3 times independently and average the results. We run each experiment for 10 epochs. The results shown in Fig. 3 suggest that $\alpha=0.2$ is a reasonable value. Therefore, we used this value for training our final multi-task model.

Table 2 shows the results of different architectures for both tasks. We can see that our model outperforms FuseNet [8] and PanNet [4] on the segmentation and pansharpening tasks, respectively. Furthermore, our multi-task model surpasses all single-task models. Multi-task learning improves results in the two tasks.

On Fig. 4, sub-figures (a-d) compare different pansharpening techniques. One can see that PanNet produces many artifacts, and that single-task outputs are blurry. On the other hand, our multi-task pansharpening output is visually closer to the ground truth. Sub-figures (e-i) illustrates tree segmentation (colored in magenta). FuseNet low $_{\text {and }}$ FuseNet skip $_{\text {. }}$

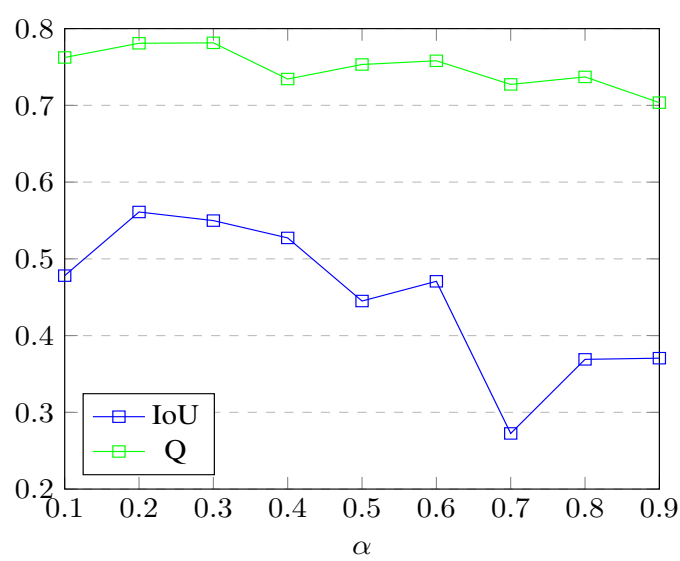

Fig. 3: Universal Image Quality Index (Q) and Intersection over Union (IoU) values on the validation set, for different $\alpha$.

produce many false negatives (many trees are missed). Our single-task and multi-task segmentation results are very close but the multi-task model produces more true positives.

\section{CONCLUSION}

In this paper, we introduced a framework that learns two different remote sensing imagery tasks simultaneously: pansharpening and segmentation. The experimental results showed that this multi-task model surpasses all single-task models (including different architectures) in both tasks. MTL proved to be able to enhance generalization performance, by representing data in a form that can be utilized by different tasks.

\section{REFERENCES}

[1] Dominique Fasbender, Julien Radoux, and Patrick Bogaert, "Bayesian data fusion for adaptable image pansharpening," IEEE Transactions on Geoscience and Remote Sensing, vol. 46, no. 6, pp. 1847-1857, 2008.

[2] Stanislas de Béthune, Fabrice Muller, and Jean-Paul Donnay, "Fusion of multispectral and panchromatic images by local mean and variance matching filtering techniques," Fusion of Earth Data, pp. 28-30, 1998.

[3] Giuseppe Masi, Davide Cozzolino, Luisa Verdoliva, and Giuseppe Scarpa, "Pansharpening by convolutional neural networks," Remote Sensing, vol. 8, no. 7, pp. 594, 2016.

[4] Junfeng Yang, Xueyang Fu, Yuwen Hu, Yue Huang, Xinghao Ding, and John Paisley, "Pannet: A deep network architecture for pan-sharpening," in Proc. IEEE Int. Conf. Comput. Vis.(ICCV), 2017, pp. 1753-1761.

[5] Emmanuel Maggiori, Yuliya Tarabalka, Guillaume 


\begin{tabular}{|c|c|c|c|c|c|}
\hline \multirow{2}{*}{ Method } & \multirow{2}{*}{ Tasks } & Seg. metrics & \multicolumn{3}{|c|}{ Pansharpening metrics } \\
\hline & & $\operatorname{IoU}[\uparrow]$ & $\mathrm{Q}[\uparrow]$ & PSNR [个] & ERGAS $[\downarrow]$ \\
\hline " FuseNet $_{\text {low }}[8]$ & "Segmentation & 0.319 & $\overline{\mathrm{N} / \mathrm{A}}$ & $\overline{\mathrm{N} / \mathrm{A}}$ & N/A \\
\hline FuseNet $_{\text {skip }}[8]$ & Segmentation & 0.325 & N/A & N/A & N/A \\
\hline Our single-task model & Segmentation & 0.599 & N/A & N/A & N/A \\
\hline PanNet [4] & Pansharpening & N/A & 0.633 & 39.6 & 19.4 \\
\hline Our single-task model & Pansharpening & N/A & 0.793 & 41.5 & 15.5 \\
\hline Our multi-task model & Pansharpening + Segmentation & 0.610 & 0.961 & 48.0 & 7.8 \\
\hline
\end{tabular}

Table 2: The results of different methods on the validation set.

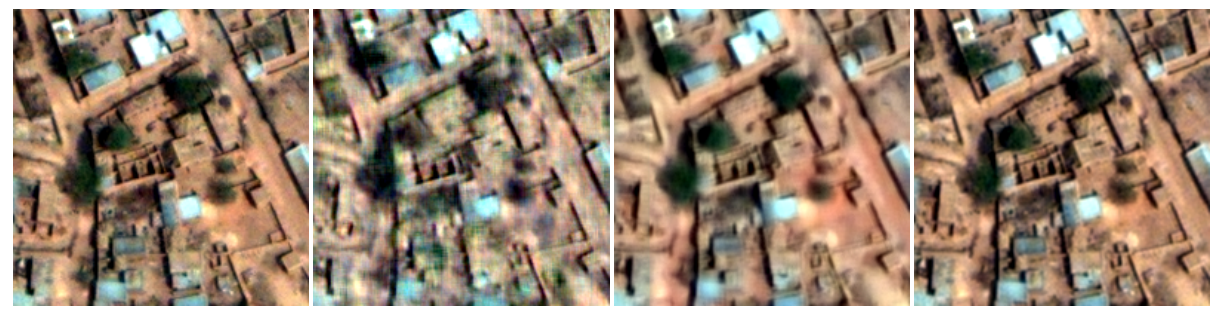

(a) Ground truth

(b) PanNet

(c) Single-task

(d) Multi-task

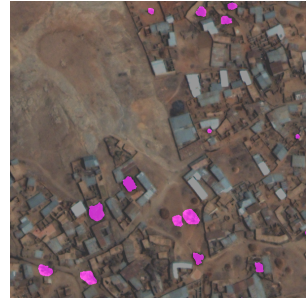

(e) Ground truth

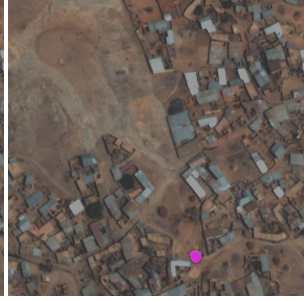

(f) FuseNet low

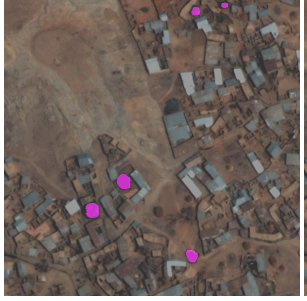

(g) FuseNet skip $_{\text {. }}$

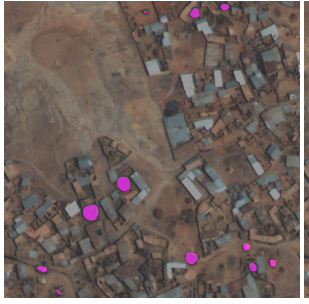

(h) Single-task

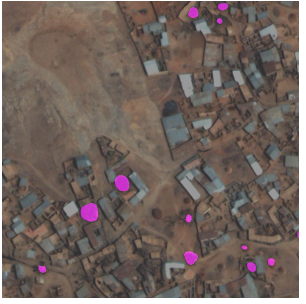

(i) Multi-task

Fig. 4: Visual results of different models on the validation set. Sub-figures (a-d) show pansharpening close-ups, while subfigures (e-i) show the segmentation of trees (colored in magenta).

Charpiat, and Pierre Alliez, "Convolutional neural networks for large-scale remote-sensing image classification," IEEE Transactions on Geoscience and Remote Sensing, vol. 55, no. 2, pp. 645-657, 2017.

[6] Olaf Ronneberger, Philipp Fischer, and Thomas Brox, "U-net: Convolutional networks for biomedical image segmentation," in International Conference on Medical image computing and computer-assisted intervention. Springer, 2015, pp. 234-241.

[7] Bohao Huang, Kangkang Lu, Nicolas Audebert, Andrew Khalel, Yuliya Tarabalka, Jordan Malof, Alexandre Boulch, Bertrand Le Saux, Leslie Collins, Kyle Bradbury, et al., "Large-scale semantic classification: outcome of the first year of inria aerial image labeling benchmark," in IGARSS, 2018.

[8] John Ray Bergado, Claudio Persello, and Alfred Stein, "Fusenet: End-to-end multispectral vhr image fusion and classification," in IGARSS, 2018, pp. 2091-2094.
[9] Jonathan Baxter, "A bayesian/information theoretic model of learning to learn via multiple task sampling," Machine learning, vol. 28, no. 1, pp. 7-39, 1997.

[10] Md Atiqur Rahman and Yang Wang, "Optimizing intersection-over-union in deep neural networks for image segmentation," in International Symposium on Visual Computing. Springer, 2016, pp. 234-244.

[11] Zhou Wang and Alan C Bovik, "A universal image quality index," IEEE signal processing letters, vol. 9, no. 3, pp. 81-84, 2002.

[12] Gabriela Csurka, Diane Larlus, Florent Perronnin, and France Meylan, "What is a good evaluation measure for semantic segmentation?.," in BMVC, 2013, vol. 27.

[13] Lucien Wald, "Quality of high resolution synthesised images: Is there a simple criterion?," in Third conference" Fusion of Earth data: merging point measurements, raster maps and remotely sensed images". SEE/URISCA, 2000, pp. 99-103. 